\title{
Clinical Study \\ Effect of Formula Feeding and Breastfeeding on Child Growth, Infant Mortality, and HIV Transmission in Children Born to HIV-Infected Pregnant Women Who Received Triple Antiretroviral Therapy in a Resource-Limited Setting: Data from an HIV Cohort Study in India
}

\author{
Gerardo Alvarez-Uria, Manoranjan Midde, Raghavakalyan Pakam, \\ Lakshminarayana Bachu, and Praveen Kumar Naik \\ Department of Infectious Diseases, Rural Development Trust Hospital, Bathalapalli, Kadiri Road, Bathalapalli 515661, India
}

Correspondence should be addressed to Gerardo Alvarez-Uria, gerardouria@gmail.com

Received 11 March 2012; Accepted 4 April 2012

Academic Editors: C. D. Berkowitz and G. D. Overturf

Copyright () 2012 Gerardo Alvarez-Uria et al. This is an open access article distributed under the Creative Commons Attribution License, which permits unrestricted use, distribution, and reproduction in any medium, provided the original work is properly cited.

We describe a programme for the prevention of mother-to-child transmission (PMTCT) of HIV that provided universal antiretroviral therapy (ART) to all pregnant women regardless of the CD4 lymphocyte count and formula feeding for children with high risk of HIV transmission through breastfeeding in a district of India. The overall rate of HIV transmission was 3.7\%. Although breastfeeding added a 3.1\% additional risk of HIV acquisition, formula-fed infants had significantly higher risk of death compared to breastfed infants. The cumulative 12 -month mortality was $9.6 \%$ for formula-fed infants versus $0.68 \%$ for breastfed infants. Anthropometric markers (weight, length/height, weight for length/height, body mass index, head circumference, mid-upper arm circumference, triceps skinfold, and subscapular skinfold) showed that formula-fed infants experience severe malnutrition during the first two months of life. We did not observe any death after rapid weaning at 5-6 months in breastfed infants. The higher-free-of HIV survival in breastfed infants and the low rate of HIV transmission found in this study support the implementation of PMTCT programmes with universal ART to all HIV-infected pregnant women and breastfeeding in order to reduce HIV transmission without increasing infant mortality in developing countries.

\section{Background}

In 2010, there were 1,490,000 HIV-infected pregnant women and 390,000 children became infected with HIV [1]. Motherto-child transmission of HIV can occur during pregnancy, during birth, or during breastfeeding. The risk of transmission is $15-30 \%$ in nonbreastfeeding populations and breastfeeding adds an additional 5-20\% risk for an overall transmission rate of $20-45 \%$ [2]. In 2010, the World Health Organization (WHO) released the guidelines on antiretroviral drugs for treating pregnant women and preventing HIV infection in infants with the goal of reducing mother-to-child transmission to less than $5 \%$ and virtually eliminating HIV infection in children by $2015[3,4]$.

Infant feeding by HIV-infected women remains a public health dilemma for developing countries. Although breastfeeding involves a considerable risk of HIV transmission, nonbreastfed infants are exposed to higher risk of death in resource-limited setting $[5,6]$. According to $2010 \mathrm{WHO}$ guidelines [4], National health authorities should decide whether health services will principally counsel and support HIV-infected women to either breastfeed and receive antiretroviral interventions or avoid breastfeeding, as the strategy that will most likely give infants the greatest chance 
of HIV-free survival. Developed countries have decided to recommend formula feeding for HIV-exposed children due to the low infant mortality and the risk of HIV transmission through breastfeeding, whereas in developing countries the health and survival benefits of breastfeeding exceed the risk of HIV transmission [7]. However, most of the evidence for recommending breastfeeding in developing countries comes from clinical trials performed in Sub-Saharan Africa. Data of formula feeding on child growth and mortality from PMTCT programmes in other continents are scarce, and some developing countries are supporting the use of formula feeding in their national PMTCT programmes [7-11].

India is the third country in the world in terms of HIV infected people, and it is estimated that 43,000 pregnant women were living with HIV in India in 2009 [12]. In this study, we investigated the effect of formula feeding and breastfeeding on mortality, growth, and HIV transmission of children enrolled in a PMTCT programme in a rural district of India.

\section{Methods}

2.1. Setting. In India, the neonatal mortality rate was 34 deaths/1000 live births and the infant mortality rate was 50 deaths/1000 live births in 2009 [13]. Andhra Pradesh is the state with the highest burden of HIV-infected people in India [14]. Anantapur is a district situated in the south border of Andhra Pradesh. Rural Development Trust (RDT) is a nongovernmental organization that has three hospitals in Anantapur. In these hospitals, medical care of HIV-infected people is provided free of cost, including medicines and consultation or admission charges.

The Vicente Ferrer HIV Cohort Study (VFHCS) is an open cohort study of all HIV-infected patients who have visited the RDT hospitals since June 2006. The characteristics of the cohort have been described elsewhere [15]. Taking into account data from the Andhra Pradesh State AIDS Control Society that estimates that $15,721 \mathrm{HIV}$-infected people are living in the district of Anantapur, the cohort is fairly representative for the district as it covers approximately $70 \%$ of all HIV-infected patients [15]. For this study, HIV-infected women from the VFHCS database who became pregnant between January 1st 2008 and December 5th 2010 were included in the analysis. The selection of patients from the database was executed in January 17th 2012.

2.2. Description of the RDT Programme for PMTCT of HIV. In order to reduce the number of children infected by HIV, RDT initiated a programme for PMTCT. In close collaboration with governmental Integrated Counselling and Testing Centres, which provided free HIV testing to all pregnant women in the district, HIV-positive pregnant women were followed by a group of 30 community health workers spread across the district. HIV-infected pregnant women initiated triple antiretroviral therapy (ART) regardless of the CD4 lymphocyte count. Delivery by caesarean section was offered to all women for reducing the risk of HIV transmission at birth, unless they had an HIV viral load below 1000 copies/mL in the third trimester of pregnancy. In women who were diagnosed with HIV during labour, a single fixed-dose combination tablet of zidovudine $300 \mathrm{mg}$, lamivudine $150 \mathrm{mg}$, and nevirapine $200 \mathrm{mg}$ was given, and caesarean section was performed only if there was no membrane rupture. Exclusive breastfeeding with rapid weaning after 5-6 months was promoted. However, women who initiated ART less than one month before delivery were counselled about the risk of HIV transmission through breastfeeding and formula feeding was offered for six months free of cost. Women who decided to give formula feeding to their babies were admitted to hospital for training on safe methods of replacement feeding such as boiling water for milk preparation and using cup feeding instead of bottle feeding $[2,16]$. ART to the mother was continued after delivery if the CD4 lymphocyte count was below 350 cells $/ \mu \mathrm{L}$ at the moment of initiating ART. In women who initiated ART with high CD4 lymphocyte count, ART was stopped either one month after stopping breastfeeding or immediately after delivery in those women who decided to give formula feeding to their children. If the ART regiment included a nonnucleoside reverse transcriptase inhibitor (nevirapine or efavirenz), two nucleoside reverse transcriptase inhibitors were continued for two extra weeks in order to reduce the risk of developing nevirapine or efavirenz resistance due to their longer life span [4].

Newborn prophylaxis was also given. Before the publication of the WHO 2010 guidelines, single (zidovudine or nevirapine) or triple (zidovudine, lamivudine, and nevirapine) drug prophylaxis in syrup form was given to newborns for 7 to 42 days according to the risk of HIV infection during delivery. After the publication of WHO 2010 guidelines, all newborns received 28 days of zidovudine syrup according to their weight.

For identifying the moment of HIV transmission, we aimed to perform HIV viral load at birth, after six weeks of delivery and after six weeks of stopping breastfeeding. Children were considered to be infected intrauterus, during delivery, or during breastfeeding if the HIV viral load was positive at birth, after six weeks of delivery, or six weeks after stopping breastfeeding, respectively. Children who did not have any HIV viral load determination were considered as HIV negative if their HIV serology was negative at any time after six months of age and were considered as HIV positive if they had a positive HIV serology after 18 months of age [17]. Measurement of HIV viral load was performed utilizing two different commercial assays, a real-time HIV1 RNA polymerase chain reaction assay (Cobas TaqMan HIV-1 v1.0, Roche Diagnostics, Mannheim, Germany) until March 2011 and a reverse transcriptase activity assay (Exavir load version 3, Cavidi, Uppsala, Sweden) since March 2011.

During the visits of children to hospitals, growth parameters such as weight, length/height, head circumference, midupper arm circumference, triceps skinfold thickness and subscapular skinfold thickness were collected. HIV-exposed children were followed up until 18 months of age with regular visits to the hospitals and with home visits by outreach workers. 
TABLE 1: Characteristics of patients enrolled in the programme before delivery.

\begin{tabular}{|c|c|}
\hline & $N(\%)$ \\
\hline Age & $23.5\left(21-25.2^{) *}\right.$ \\
\hline Height & $152(148-160)^{*}$ \\
\hline Underweight (BMI < 18.5) & $122(39)$ \\
\hline First pregnancy & $173(51.49)$ \\
\hline \multicolumn{2}{|l|}{ Education } \\
\hline Higher & $13(3.88)$ \\
\hline Secondary & $128(38.21)$ \\
\hline Primary & $48(14.33)$ \\
\hline No education & $146(43.58)$ \\
\hline Smoker & $1(0.003)$ \\
\hline \multicolumn{2}{|c|}{ CD4 lymphocyte count (cells/ $\mu \mathrm{L}$ ) } \\
\hline$<200$ & $29(8.76)$ \\
\hline $200-350$ & $96(29)$ \\
\hline $350-500$ & $102(30.82)$ \\
\hline$>500$ & $104(31.42)$ \\
\hline \multicolumn{2}{|l|}{ Time on ART before delivery } \\
\hline$<1$ day & $23(6.91)$ \\
\hline $1-30$ days & $53(15.92)$ \\
\hline 31-60 days & $48(14.41)$ \\
\hline $61-90$ days & $54(16.22)$ \\
\hline $91-120$ days & $40(12.01)$ \\
\hline$>120$ days & $115(34.53)$ \\
\hline \multicolumn{2}{|l|}{ ART regimen } \\
\hline $\mathrm{AZT}+3 \mathrm{TC}+\mathrm{NVP}$ & $148(44.05)$ \\
\hline $\mathrm{AZT}+3 \mathrm{TC}+\mathrm{EFV}$ & $68(20.24)$ \\
\hline $\mathrm{AZT}+3 \mathrm{TC}+\mathrm{NFV}$ & $35(10.42)$ \\
\hline $\mathrm{D} 4 \mathrm{~T}+3 \mathrm{TC}+\mathrm{NVP}$ & $39(11.61)$ \\
\hline $\mathrm{D} 4 \mathrm{~T}+3 \mathrm{TC}+\mathrm{EFV}$ & $19(5.65)$ \\
\hline $\mathrm{D} 4 \mathrm{~T}+3 \mathrm{TC}+\mathrm{NFV}$ & $16(4.76)$ \\
\hline $\mathrm{TDF}+3 \mathrm{TC} / \mathrm{FTC}+\mathrm{LPVr}$ & $5(1.49)$ \\
\hline $\mathrm{AZT}+3 \mathrm{TC}+\mathrm{LPVr}$ & $4(1.19)$ \\
\hline $\mathrm{d} 4 \mathrm{~T}+3 \mathrm{TC}+\mathrm{LPVr}$ & $1(0.3)$ \\
\hline $\mathrm{TDF}+3 \mathrm{TC}+\mathrm{AZT}$ & $1(0.3)$ \\
\hline Home delivery & $24(7.38)$ \\
\hline Caesarean section & $125(39.18)$ \\
\hline \multicolumn{2}{|l|}{ Newborn prophylaxis } \\
\hline $\mathrm{AZT}$ & $229(73.63)$ \\
\hline $\mathrm{AZT}+3 \mathrm{TC}+\mathrm{NVP}$ & $59(18.97)$ \\
\hline NVP & $15(4.82)$ \\
\hline None & $8(2.57)$ \\
\hline \multicolumn{2}{|l|}{ Type of feeding } \\
\hline Formula & $159(51.29)$ \\
\hline Breast & $148(47.74)$ \\
\hline Mixed & $3(0.97)$ \\
\hline
\end{tabular}

${ }^{*}$ Median (interquartile range). BMI: body mass index; ART: antiretroviral treatment; AZT: zidovudine; 3TC: lamivudine; NVP, and nevirapine; EFV, efavirenz; d4T: stavudine; NFV: nelfinavir; TDF: tenofovir; FTC: emtricitabine; LPVr: lopinavir-ritonavir. Missing values for each variable were not included.
2.3. Statistical Analysis. Statistical analysis was performed using Stata Statistical Software (Stata Corporation, Release 11, College Station, Texas, USA). Confidence intervals for proportions were estimated utilizing the Wilson method [18]. We used Kaplan-Meier curves and log-rank test for comparing mortality in children who received breastfeeding or formula feeding. Time was measured from birth to death or the last visit date. For calculating Z-scores of anthropometric indicators according to WHO child growth standards for age and sex, we utilized the WHO macro for Stata (igrowup_standard.ado) [19]. WHO child growth standards were calculated from a group of selected children from different parts of the world who received optimal nutrition according to WHO standards: no known health or environmental constraints to growth, mothers willing to follow specific feeding recommendations (i.e., exclusive or predominant breastfeeding for at least four months, introduction of complementary foods by the age of six months and continued partial breastfeeding up to at least 12 months), no maternal smoking before and after delivery, single term birth, and absence of significant morbidity [20]. Among all anthropometric markers, we selected severe underweight (weight-for-age $<-3 \mathrm{Z}$-scores) because it has demonstrated to be a good indicator for predicting mortality in infants from India, especially in infants aged less than six months [21]. The study was approved by the ethical committee of the RDT Institutional Review Board.

\section{Results}

During the study period, $391 \mathrm{HIV}$-infected pregnant women were identified and $336(85.9 \%)$ entered in the PMTCT programme before delivery. Of the 55 pregnancies that did not enter in the programme before delivery, five children were lost to follow up and 12 out of 50 (24\%) were HIV infected although $29(58 \%)$ of them received formula feeding.

Characteristics of the 336 women who entered in the programme before delivery are described in Table 1 . The median age was 23 years, and the median height was $152 \mathrm{~cm}$. The proportion of underweight in mothers was 39\%, and approximately half of them were primipara. Almost 40\% were illiterate and only one was smoker. The proportion of pregnant women with CD4 lymphocyte count below 200 and 350 cells $/ \mu \mathrm{L}$ were $9 \%$ and $29 \%$, respectively. Zidovudine was used in $76 \%$ of women, and nevirapine was used more frequently than efavirenz. Near 7\% initiated ART at the same day of delivery. Viral load in the third trimester was available for 199 women and $175(88 \%)$ of them had a viral load below 1000 copies $/ \mathrm{mL}$. Seven percent of women delivered at home, and caesarean section was performed in 39\% of the cases. Zidovudine syrup was used more commonly than other newborn prophylaxis regimens, and $51 \%$ of children received formula feeding.

In Figure 1, we present a flow diagram of the outcomes of all HIV-positive pregnancies that entered in the PMTCT programme before delivery. Of the three pregnant women who had spontaneous abortion, two started ART (zidovudine, 


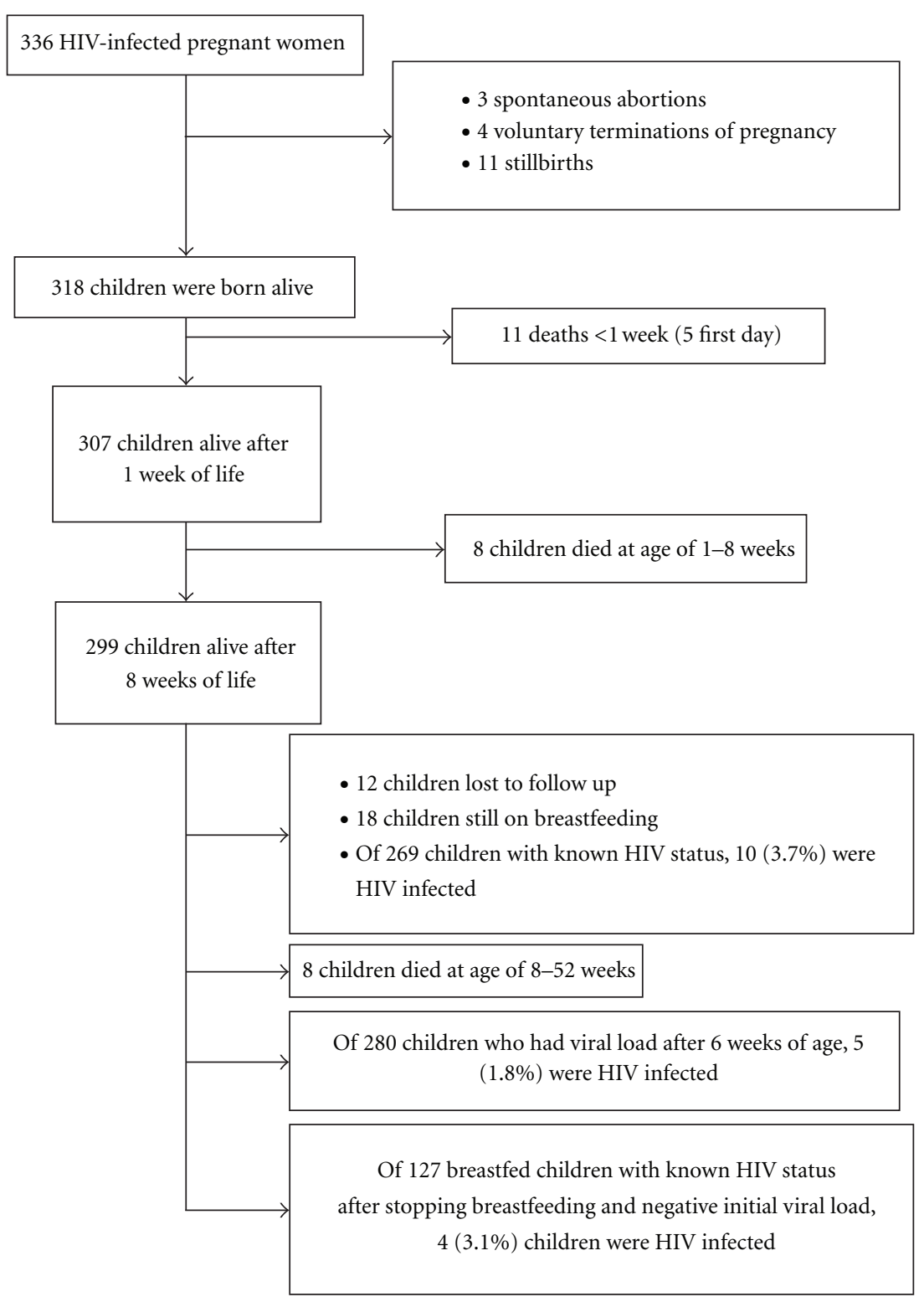

FIGURE 1: Flowchart of the outcomes of the study.

lamivudine, and nevirapine) before having the abortion. All voluntary terminations of pregnancy occurred between the third and the fifth month of pregnancy. Of the 11 children who died during the first week of life, weight was recorded in five and in four of them the birthweight was less than $1.5 \mathrm{~kg}$. No mother died during pregnancy or during delivery. The perinatal mortality rate was 22/329 (67 deaths/1000 total births, 95\% confidence interval (CI) 45 to 99), the neonatal mortality rate was $12 / 318$ (38 deaths/1000 live births, 95\% CI 22 to 65), and the infant mortality rate was $27 / 318$ (85 deaths/1000 live births, 95\% CI 59 to 121$)$. The overall transmission of HIV was $3.7 \%$ (95\% CI, 2 to 6.7$)$, although $12(4 \%)$ were lost to follow up and 18 who had a negative viral load after six weeks of life were still on breastfeeding at the end of the study period. Antenatal and perinatal preventive measures were able to reduce the rate of HIV transmission to $1.8 \%$ (95\% CI, 0.7 to 4.1 ) in those children who had a viral load determination after the age of six weeks. Of those breastfed children who had a negative viral load determination after aged six weeks, 3.1\% (95\% CI, 1.2 to 7.8 ) acquired HIV during breastfeeding. In this group, ART was initiated at least one month before delivery in all but one of the cases $(99.2 \%)$. The characteristics of the ten children who became HIV infected are presented in Table 2.

To study the effect of the type of feeding on infant mortality we excluded children who died during the first week of life to avoid causes of the death related to perinatal complications [22, 23]. Kaplan-Meier survival curves of infants by type of feeding are presented in Figure 2. Three quarters of deaths (12 out of 16) occurred during the first trimester of life. Compared to breastfed children, formulafed children had a crude hazard ratio for death of 15.2 


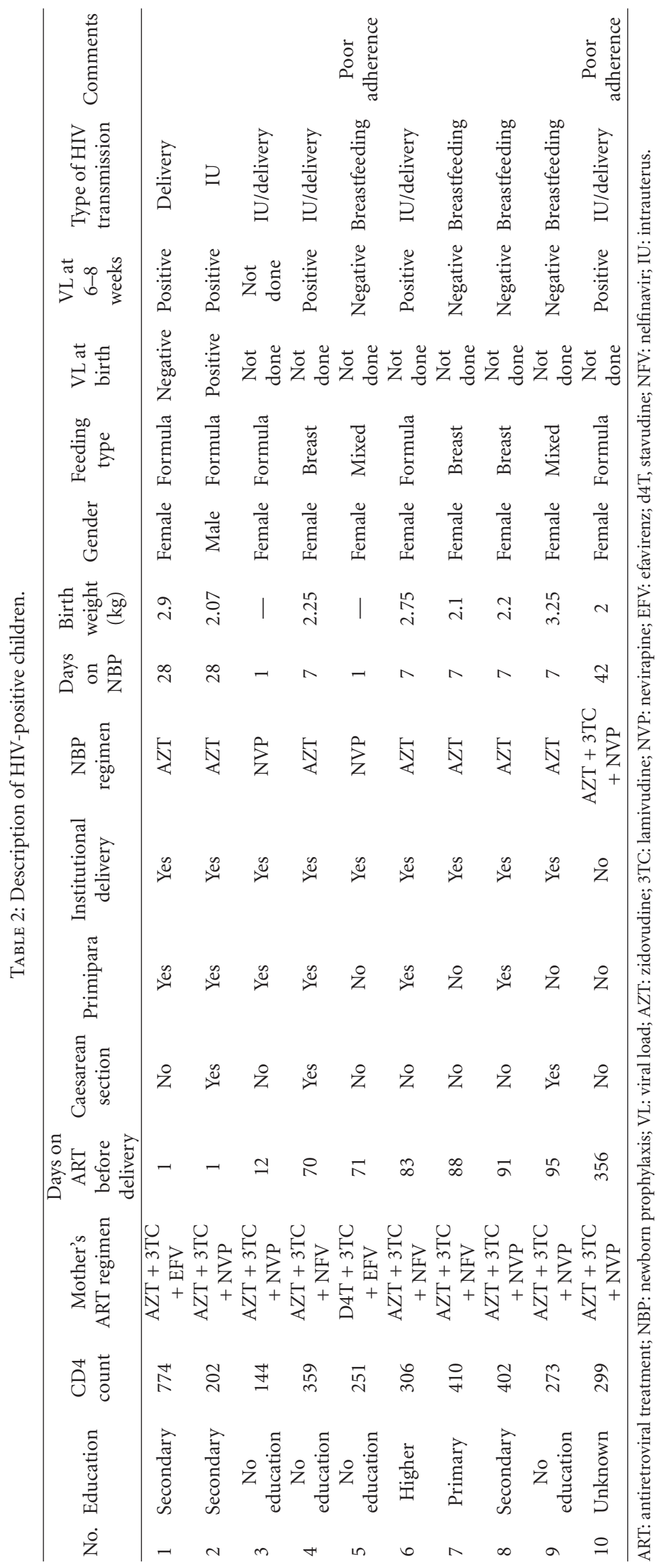



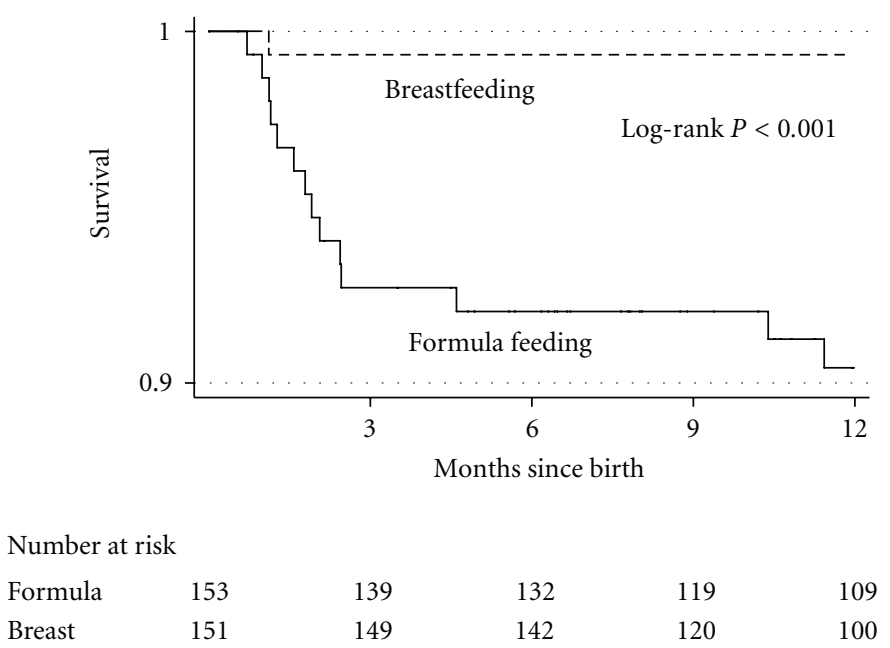

FIgURE 2: Kaplan-Meier survival curves of infants by type of feeding.

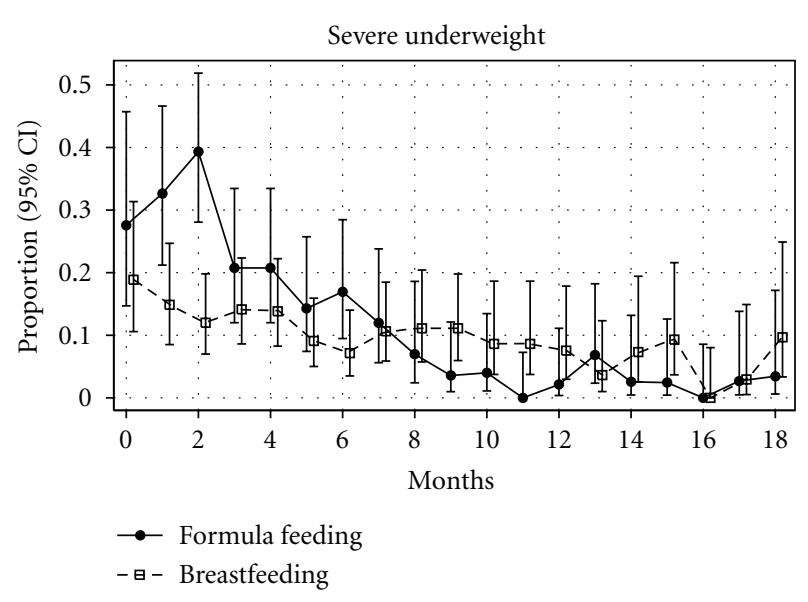

Figure 3: Proportion of children with severe underweight $(<-3 \mathrm{Z}$ score) and $95 \%$ confidence interval during the first 18 months of life by type of feeding.

(95\% CI, 2 to 114.8 ) ( $P=0.008)$. In a multivariable analysis, the hazard ratio for death in formula-fed children was 13.5 (95\% CI, 1.7 to 108.2$)(P=0.014)$ adjusted by low birth weight $(<2.5 \mathrm{~kg})$, low CD4 lymphocyte count of the mother $(<200$ cells $/ \mu \mathrm{L})$, home delivery, illiteracy of the mother and less than one month on ART before delivery [24]. The cumulative mortality at three months was 7.3\% (95\% CI, 4.1 to 12.7$)$ for formula-fed infants and $0.66 \%(95 \% \mathrm{CI}$, 0.1 to 4.6 ) for breastfed infants. The cumulative mortality at 12 months was $9.6 \%$ (95\% CI, 6.7 to 18.5$)$ for formula fed infants and $0.68 \%(95 \% \mathrm{CI}, 0.1$ to 4.7$)$ for breastfed infants. Free-of-HIV survival was also significantly higher in breastfed children $(126 / 131,96.2 \%)$ than in formula-fed children $(130 / 151,86.1 \%)(P=0.003)$. When questioning mothers about the symptoms of the child before death, eight reported gastrointestinal symptoms, three reported fever, three reported seizures, one reported respiratory symptoms, and one reported low consciousness.
The proportion of children with severe underweight by type of feeding during the first 18 months of life is presented in Figure 3. The proportion of infants with severe underweight who received formula feeding was higher in the first two months of life, reaching a pick of $40 \%$ at two months, but it was reduced notably afterwards. The proportion of infants with severe underweight who received breastfeeding experienced progressive reduction over time. The mean and 95\% CI of anthropometric markers (weight, length/height, body mass index, weight for length/height, head circumference, mid-upper arm circumference, triceps skinfold thickness, and subscapular skinfold thickness) for age and sex by type of feeding during the first 18 months of life are presented in Figure 4. Whereas biomarkers of breastfed infants remained more or less stable over time, formula-fed infants experienced a rapid decline during the first two months of life and a rapid improvement after the third month of life, achieving similar or superior values to breastfed infants after the ninth month of life.

\section{Discussion}

In this study, the use of formula feeding was associated with increased risk of mortality and lower HIV-free survival compared to breastfeeding. In India, $98 \%$ of children are breastfed and it is estimated that $54 \%$ of all deaths before age of five years are related to malnutrition [25]. The introduction of formula feeding for averting the transmission of HIV imposes an additional handicap to an already malnourished population. As it is shown in Figures 3 and 4, formulafed children experience an acute decline in anthropometric markers during the first two months of life, when most of deaths occurred. Our findings are in accordance with the results of other studies performed in resource-limited settings that have shown an increased morbidity and mortality in HIV-exposed children who received formula feeding $[7,26,27]$. Also in India, HIV-exposed children from a large 


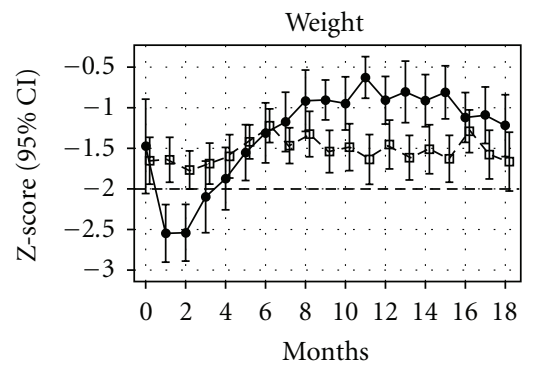

(a)

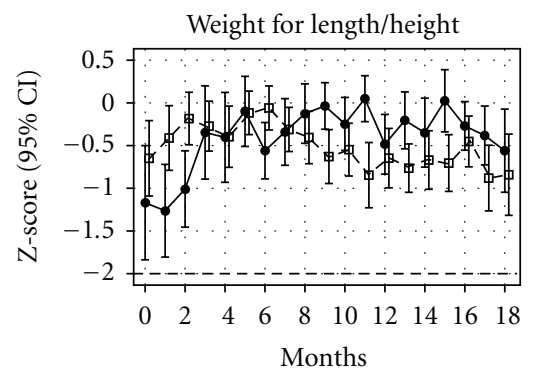

(d)

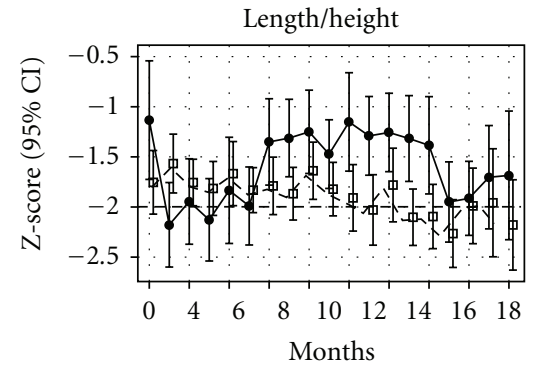

(b)

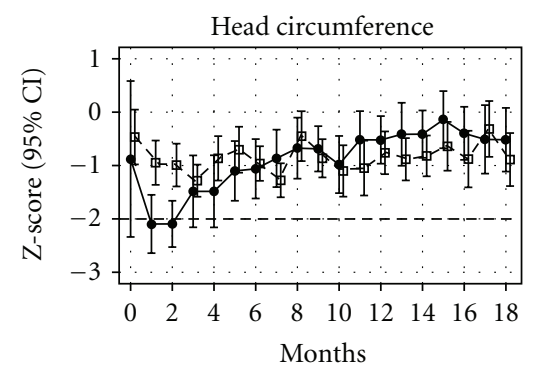

(e)

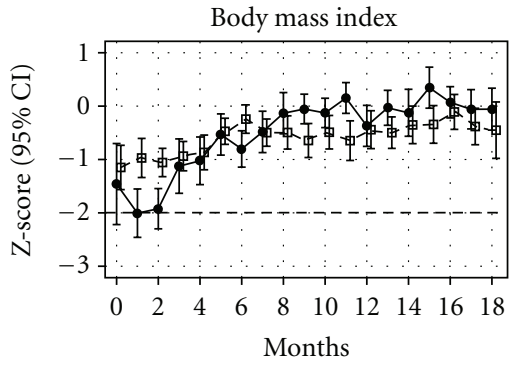

(c)

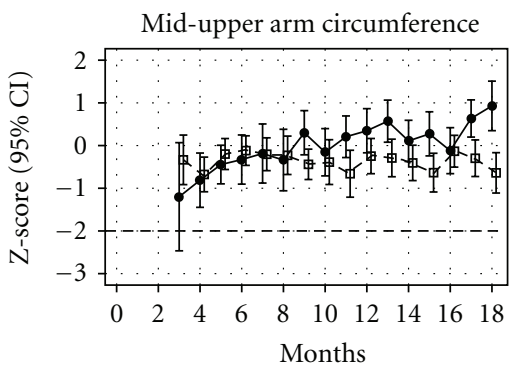

(f)

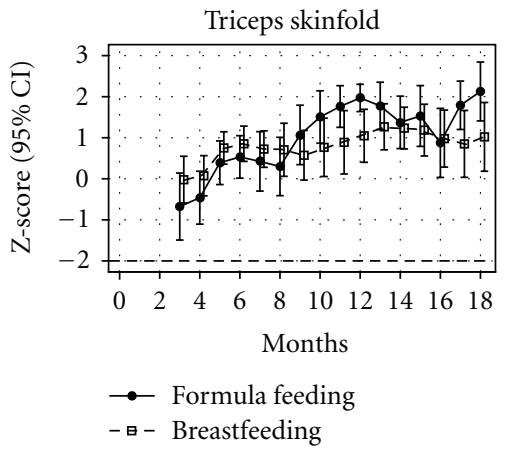

(g)

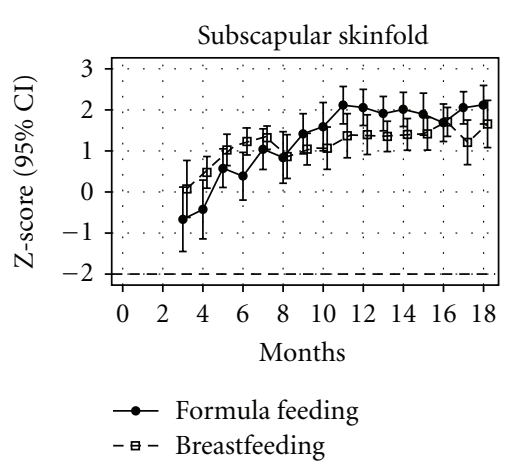

(h)

FIGURE 4: Mean and 95\% confidence interval of anthropometric markers during the first 18 months of life by type of feeding adjusted by age and sex.

government hospital who were formula fed had an increased risk of hospitalization [28].

Although the overall proportion of HIV-infected children was 3.7\%, prenatal and perinatal interventions achieved a HIV transmission rate of $1.8 \%$, which is similar to the rate of HIV transmission in developed countries where the majority of children receive formula feeding [29]. The rate of HIV transmission due to breastfeeding was 3.1\%, which is slightly higher than the rate reported in clinical trials in Africa [30, 31].

The breastfed infants were heavier and taller during the first two months, but lighter and shorter thereafter. Looking at the graph of weight for length/height (a marker of acute malnutrition) in Figure 4, we can observe a decline after stopping breastfeeding at six months of age compared to WHO growth standards, which were created from a population of infants who were breastfed at least until the age of 12 months [32]. However, we did not observe an increased mortality in the breastfed group after weaning at age 5-6 months as it was seen in clinical trials in Africa with abrupt weaning at the age of four months [33, 34]. This observation is important because the 2010 WHO guidelines recommend breastfeeding until the age of 12 months [4], but the initiation of mixed feeding can increase the risk of HIV transmission [24]. New studies comparing morbidity, mortality, and HIV transmission in children who receive breastfeeding for six or twelve months may clarify this matter.

The proportion of women with underweight was similar to the proportion of women from rural areas with underweight described in the National Family Health Survey, a large-scale survey conducted in a representative sample of households throughout India [25]. In the same study, it was observed that $48 \%$ of children under five years had stunting (length/height for age $<-2 \mathrm{Z}$-scores), $20 \%$ had wasting (weight for length/height $<-2$ Z-scores) and $43 \%$ had underweight (weight for age $<-2 \mathrm{Z}$-scores). These data can explain the low values in length/height, weight for length/height and weight observed in Figure 4. 
However, anthropometric markers related to fat deposits such as triceps and subscapular skinfold thickness had similar or even higher values than WHO growth standards. These differences may be explained by the low quantity of proteins in the diet in poor and rural areas of India $[15,25]$.

The study has some limitations. The mother's ART was initiated at least one month before delivery in almost all children who received breastfeeding. It is possible that the rate of HIV transmission due to breastfeeding will rise if breastfeeding is encouraged to mothers who initiate ART late in pregnancy or during labour. New studies are needed to assess HIV-free survival if breastfeeding is universally recommended regardless of the time on ART before delivery.

\section{Conclusions}

Even in rural areas of India, universal ART to HIV-infected pregnant women is able to reduce mother-to-child transmission to less than $5 \%$. Although breastfeeding can increase the rate of HIV transmission, formula feeding produces malnutrition during the first months of life, increases mortality and, therefore, has lower HIV-free survival compared to breastfeeding. The results of this study confirm the results of clinical trials performed in Sub-Saharan Africa and support the implementation of universal ART regardless of CD4 count to all HIV-infected pregnant women and breastfeeding for all HIV-exposed children in order to achieve WHO goals of elimination of paediatric HIV without increasing infant mortality in developing countries with high levels of malnutrition.

\section{Conflict of Interests}

The authors declare that they have no competing interests.

\section{Acknowledgments}

No funding was received for this study. The authors would like to thank Claudia Fortuny for her help in designing the RDT programme to prevent vertical transmission of HIV.

\section{References}

[1] WHO, UNICEF, and UNAIDS, "Progress report 2011: Global HIV/AIDS response," 2011.

[2] K. M. De Cock, M. G. Fowler, E. Mercier et al., "Prevention of mother-to-child HIV transmission in resource-poor countries: translating research into policy and practice," Journal of the American Medical Association, vol. 283, no. 9, pp. 1175$1182,2000$.

[3] World Health Organization, "Global health sector strategy on HIV/AIDS 2011-2015,” 2011.

[4] World Health Organization, "Antiretroviral drugs for treating pregnant women and preventing HIV infection in infants: towards universal access," 2010.

[5] R. E. Black, S. S. Morris, and J. Bryce, "Where and why are 10 million children dying every year?" The Lancet, vol. 361, no. 9376, pp. 2226-2234, 2003.
[6] C. G. Victora and A. J. D. Barros, "Effect of breastfeeding on infant and child mortality due to infectious diseases in less developed countries: a pooled analysis," The Lancet, vol. 355, no. 9202, pp. 451-455, 2000.

[7] L. Kuhn and G. Aldrovandi, "Survival and health benefits of breastfeeding versus artificial feeding in infants of hivinfected women: developing versus developed world," Clinics in Perinatology, vol. 37, no. 4, pp. 843-862, 2010.

[8] H. Coovadia, "Current issues in prevention of mother-to-child transmission of HIV-1," Current Opinion in HIV and AIDS, vol. 4, no. 4, pp. 319-324, 2009.

[9] T. Doherty, D. Sanders, A. Goga, and D. Jackson, "Implications of the new WHO guidelines on HIV and infant feeding for child survival in South Africa," Bulletin of the World Health Organization, vol. 89, no. 1, pp. 62-67, 2011.

[10] N. Phanuphak, R. Lolekha, K. Chokephaibulkit et al., "Thai national guidelines for the prevention of motherto-child transmission of HIV: March 2010," Asian Biomedicine, vol. 4, no. 4, pp. 529-540, 2010.

[11] L. H. Matida, M. H. Da Silva, A. Tayra et al., "Prevention of mother-to-child transmission of HIV in São Paulo State, Brazil: an update," AIDS, vol. 19, no. 4, supplement, pp. S37S41, 2005.

[12] WHO, UNAIDS, and UNICEF, "Scaling up priority HIV/AIDS interventions in the health sector," 2010.

[13] UNICEF-India-Statistics, http://www.unicef.org/infobycountry/india_statistics.html.

[14] UNAIDS/WHO, "Country Progress Report. UNGASS. India, March 2010,” 2010.

[15] G. Alvarez-Uria, M. Midde, R. Pakam, and P. K. Naik, "Gender differences, routes of transmission, socio-demographic characteristics and prevalence of HIV related infections of adults and children in an HIV cohort from a rural district of India," Infectious Disease Reports, vol. 4, pp. 66-70, 2012,.

[16] World Health Organization, "HIV and infant feeding counselling tools: reference guide," 2005.

[17] A. Schroder-Lorenz and L. Rensing, "Circadian changes in protein syhtetic rate and protein phosphorylation in cell-free extracts of Gonyaulax poledra," Planta, vol. 170, pp. 7-13.

[18] A. Agresti and B. A. Coull, "Approximate is better than "exact" for interval estimation of binomial proportions," American Statistician, vol. 52, no. 2, pp. 119-126, 1998.

[19] "WHO Anthro (version 3.2.2, January 2011) and macros," http://www.who.int/childgrowth/software/en/.

[20] M. de Onis, C. Garza, C. G. Victora, A. W. Onyango, E. A. Frongillo, and J. Martines, "The WHO Multicentre Growth Reference Study: planning, study design, and methodology," Food and Nutrition Bulletin, vol. 25, no. 1, pp. S15-S26, 2004.

[21] L. Vesel, R. Bahl, J. Martines et al., "Use of new World Health Organization child growth standards to assess how infant malnutrition relates to breastfeeding and mortality," Bulletin of the World Health Organization, vol. 88, no. 1, pp. 39-48, 2010.

[22] J. Zupan, "Perinatal mortality in developing countries," The New England Journal of Medicine, vol. 352, no. 20, pp. 20472048, 2005.

[23] K. M. Edmond, C. Zandoh, M. A. Quigley, S. Amenga-Etego, S. Owusu-Agyei, and B. R. Kirkwood, "Delayed breastfeeding initiation increases risk of neonatal mortality," Pediatrics, vol. 117, no. 3, pp. e380-e386, 2006.

[24] H. M. Coovadia, N. C. Rollins, R. M. Bland et al., "Motherto-child transmission of HIV-1 infection during exclusive breastfeeding in the first 6 months of life: an intervention 
cohort study," The Lancet, vol. 369, no. 9567, pp. 1107-1116, 2007.

[25] F. Arnold, "Sciences II for P, International M: Nutrition in India," International Institute for Population Sciences, 2009.

[26] I. Thior, S. Lockman, L. M. Smeaton et al., "Breastfeeding plus infant zidovudine prophylaxis for 6 months vs formula feeding plus infant zidovudine for 1 month to reduce motherto-child HIV transmission in Botswana-a randomized trial: the Mashi study," Journal of the American Medical Association, vol. 296, no. 7, pp. 794-805, 2006.

[27] E. M. Obimbo, D. A. Mbori-Ngacha, J. O. Ochieng et al., "Predictors of early mortality in a cohort of human immunodeficiency virus type 1-infected African children," Pediatric Infectious Disease Journal, vol. 23, no. 6, pp. 536-543, 2004.

[28] M. A. Phadke, B. Gadgil, K. E. Bharucha et al., "Replacementfed infants born to HIV-infected mothers in India have a high early postpartum rate of hospitalization," Journal of Nutrition, vol. 133, no. 10, pp. 3153-3157, 2003.

[29] M. G. Fowler, M. A. Lampe, D. J. Jamieson, A. P. Kourtis, and M. F. Rogers, "Reducing the risk of mother-to-child human immunodeficiency virus transmission: past successes, current progress and challenges, and future directions," American Journal of Obstetrics and Gynecology, vol. 197, no. 3, pp. S3S9, 2007.

[30] J. McIntyre, "Use of antiretrovirals during pregnancy and breastfeeding in low-income and middle-income countries," Current Opinion in HIV and AIDS, vol. 5, no. 1, pp. 48-53, 2010.

[31] R. L. Shapiro, M. D. Hughes, A. Ogwu et al., "Antiretroviral regimens in pregnancy and breast-feeding in Botswana," The New England Journal of Medicine, vol. 362, no. 24, pp. 22822294, 2010.

[32] S. Arpadi, A. Fawzy, G. M. Aldrovandi et al., "Growth faltering due to breastfeeding cessation in uninfected children born to HIV-infected mothers in Zambia," American Journal of Clinical Nutrition, vol. 90, no. 2, pp. 344-353, 2009.

[33] M. P. Fox, D. Brooks, L. Kuhn et al., "Reduced mortality associated with breast-feeding-acquired HIV infection and breast-feeding among HIV-infected children in Zambia," Journal of Acquired Immune Deficiency Syndromes, vol. 48, no. 1, pp. 90-96, 2008.

[34] L. Kuhn, G. M. Aldrovandi, M. Sinkala et al., "Effects of early, abrupt weaning on HIV-free survival of children in Zambia," The New England Journal of Medicine, vol. 359, no. 2, pp. 130 $141,2008$. 


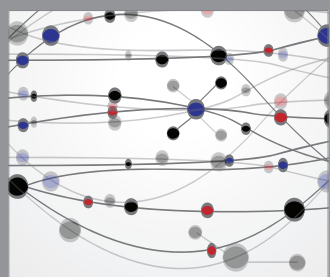

The Scientific World Journal
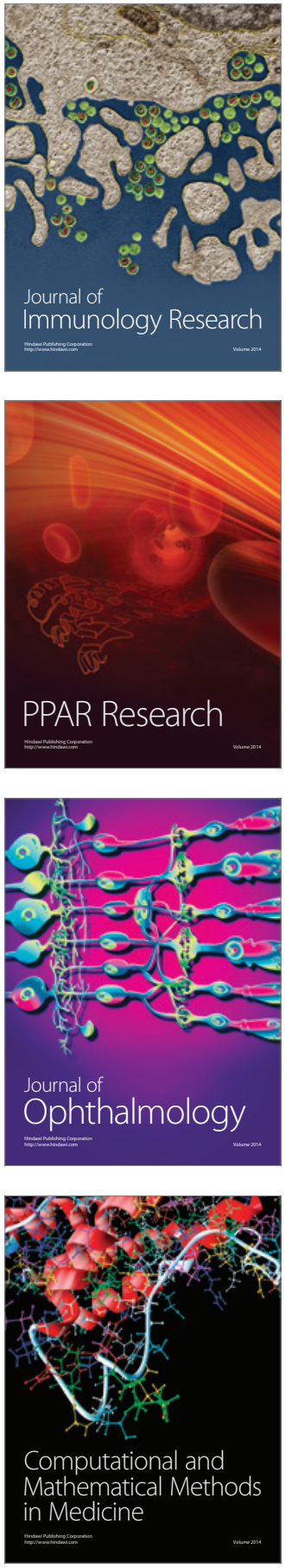

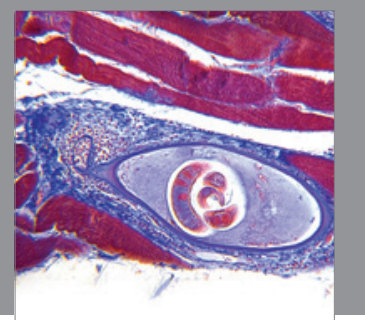

Gastroenterology

Research and Practice
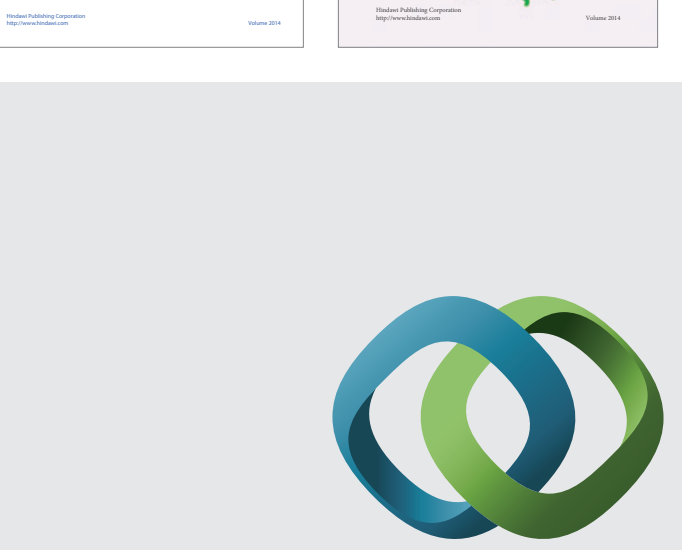

\section{Hindawi}

Submit your manuscripts at

http://www.hindawi.com
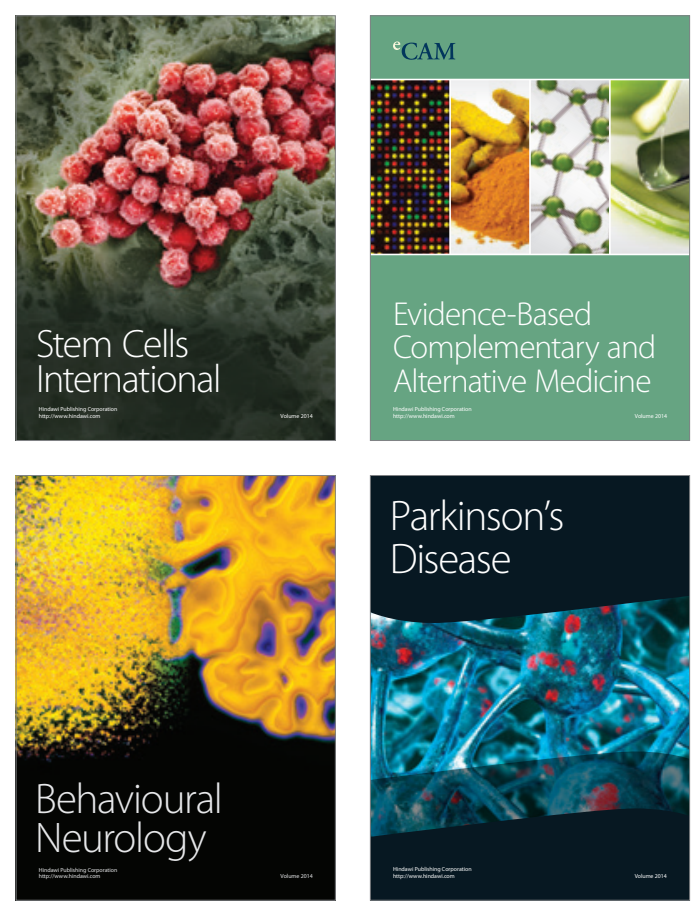

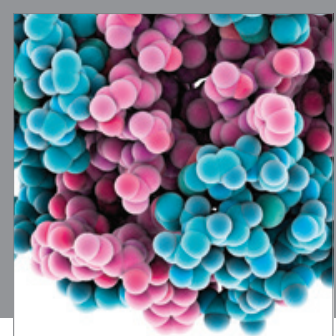

Journal of
Diabetes Research

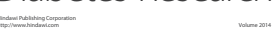

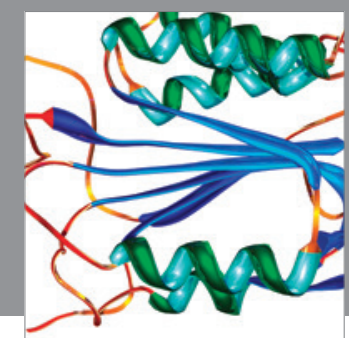

Disease Markers
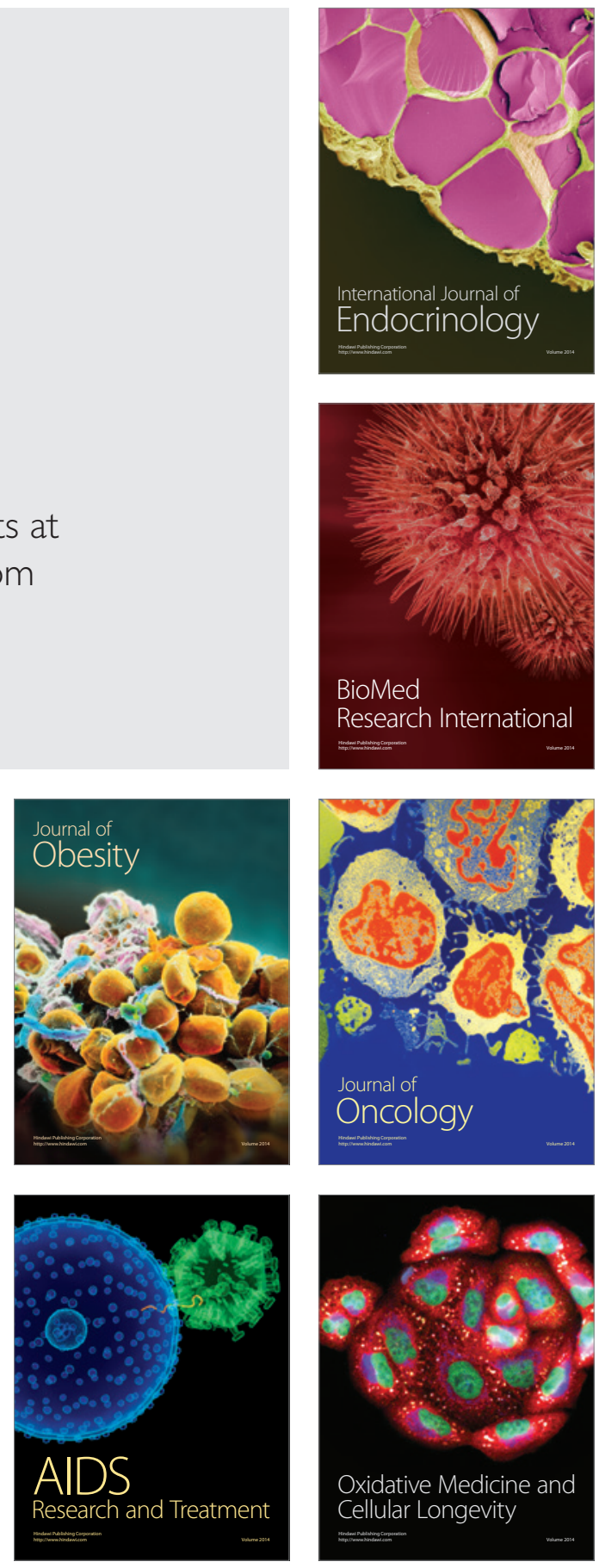